\title{
An Improved Atmospheric Correction Algorithm for Hyperspectral Remotely Sensed Imagery
}

\author{
Shunlin Liang, Senior Member, IEEE, and Hongliang Fang, Student Member, IEEE
}

\begin{abstract}
There is an increased trend toward quantitative estimation of land surface variables from hyperspectral remote sensing. One challenging issue is retrieving surface reflectance spectra from observed radiance through atmospheric correction, most methods for which are intended to correct water vapor and other absorbing gases. In this letter, methods for correcting both aerosols and water vapor are explored. We first apply the cluster matching technique developed earlier for Landsat-7 ETM+ imagery to Airborne Visible/Infrared Imaging Spectrometer (AVIRIS) data, then improve its aerosol estimation and incorporate a new method for estimating column water vapor content using the neural network technique. The improved algorithm is then used to correct Hyperion imagery. Case studies using AVIRIS and Hyperion images demonstrate that both the original and improved methods are very effective to remove heterogeneous atmospheric effects and recover surface reflectance spectra.
\end{abstract}

Index Terms-Aerosol, Airborne Visible/Infrared Imaging Spectrometer (AVIRIS), atmospheric correction, Hyperion, hyperspectral, imaging spectroscopy, remote sensing, water vapor.

\section{INTRODUCTION}

H YPERSPECTRAL remote sensing, or imaging spectroscopy, is a relatively new technology, beginning in the mid-1980s. Multispectral datasets are usually composed of about 5-10 bands of relatively large bandwidths ( $>10 \mathrm{~nm}$ ), whereas hyperspectral datasets are generally composed of about 100-200 spectral bands of relatively narrow $(5-10 \mathrm{~nm})$ bandwidth. The Airborne Visible/Infrared Imaging Spectrometer (AVIRIS) [1] is a typical airborne hyperspectral system. The first civilian spaceborne hyperspectral sensor, Hyperion [2], on the National Aeronautics and Space Administration (NASA) Earth Observing 1 (EO-1) satellite [3], has collected considerable worldwide data.

Many applications can take advantage of the hyperspectral remotely sensed signatures. Increasing number of quantitative techniques have been used to estimate land-surface, bio-, and geophysical variables from surface reflectance [4]. The procedure converting at-sensor radiance to surface reflectance is termed atmospheric correction.

There is a relatively long history of quantitative atmospheric correction of remotely sensed imagery [5], and many of the recent algorithms are reviewed in the new book [4]. Besides many statistical methods, most physically based atmospheric correction methods for heterogeneous aerosol effects rely heavily on

Manuscript received June 25, 2003; revised January 5, 2004. This work was supported in part by the National Aeronautics and Space Administration under EO1 Grant NCC5462.

The authors are with the Department of Geography, University of Maryland, College Park, MD 20742 USA (e-mail: sliang@ geog.umd.edu).

Digital Object Identifier 10.1109/LGRS.2004.824747 these remote sensing signatures: spatial, spectral, angular, temporal, and polarization.

- The spatial signatures include spatial contrast of reflectance [6], [7], e.g., matching histograms of hazy and clear regions [8] and the cluster matching technique that relies on individual clusters in both hazy and clear regions [9], [10].

- Spectral signatures are based on multispectral bands and used by most methods. For example, in cluster-matching [9], the near-infrared (NIR) bands are used to identify the clusters for correcting the visible bands. The widely used "dark-object" methods (e.g., [11]-[13]) are based on linear relationships of surface reflectance between one shortwave-infrared band (around $2.1 \mathrm{~m}$ ) and blue and red visible bands.

- Angular signatures, derived from multiangular observations, have several methods for estimating aerosol properties-such as the Multi-angle Imaging Spectroradiometer (MISR) [14], and Along Track Scanning Radiometer (ATSR) [15].

- Temporal signatures have recently found use in estimating aerosol properties from Geostationary Operational Experimental Satellite (GOES) [16] and MODIS (ModerateResolution Imaging Spectroradiometer) [17], and other datasets based on a sequence of observations of the same pixel.

- Polarization signatures are mainly from Polarization and Directionality of the Earth's Reflectance (POLDAR) data [18].

If we know the atmospheric properties, correcting atmospheric effects is relatively easy. However, obtaining accurate ancillary information on atmospheric properties is often difficult. The most practical way is to estimate directly from imagery. There are at least two primary atmospheric variables that need to be estimated: aerosol optical depth and water vapor content. Most methods for hyperspectral imagery emphasize the estimation of water vapor and other gases from images themselves [1], [19]-[21] but require aerosol properties as separate inputs. Our main objective is to develop a methodology for estimation of heterogeneous aerosol and water vapor contents and then to retrieve surface reflectance from hyperspectral imagery (e.g., AVIRIS and Hyperion).

This letter includes two parts: first, we apply the cluster matching technique [9], [10] to correct AVIRIS data. The outline and case study are given in the next section. Second, we improve the cluster matching algorithm particularly for hyperspectral imagery with an enhanced aerosol estimation component and a new water vapor estimation algorithm. The 
improved algorithm and the case studies are presented in Section III. A short section of summary and conclusions is presented at the end.

\section{Cluster Matching Algorithm FOR AVIRIS}

AVIRIS, which delivers calibrated images of upwelling spectral radiance in 224 contiguous spectral bands with wavelengths from 400-2500 nm, has been flown on two aircraft platforms: a NASA ER-2 jet and the Twin Otter turboprop. The ER-2 flies at $20 \mathrm{~km}$ above sea level and produces imagery at a $20-\mathrm{m}$ spatial resolution. The Twin Otter flies at $4-\mathrm{km}$ above-ground level and produces imagery at a spatial resolution of meters. AVIRIS has overflown North America, South America, and Europe. Refer to the literature for a detailed description of the AVIRIS instrument [1].

\section{A. Extended Algorithm}

In this experiment, we extended the cluster matching technique developed for multispectral remotely sensed data [9], [10] to correct AVIRIS data. This algorithm is based on the following assumptions: 1) NIR bands have less aerosol contamination and can classify all ground types into clusters and 2) there are clear regions over the study area whose aerosol optical depths can be easily determined by assuming an aerosol type and loading, and the same cluster in hazy or clear regions has similar surface reflectance values. Clusters represent surface cover types with unique spectral signatures (soil, vegetation canopy, and water.) Algorithm details are available in the original papers and we outline the basic procedures in conjunction with AVIRIS data here, which basically include the following six steps.

Step 1) Determining hazy regions from shortwave bands with unsupervised clustering analysis. In this spectral region, signals received by the sensor are dominated by atmospheric scattering. If surface elevation is semi-homogenous, variations are caused by aerosols. A visual examination of distribution generates a mask of hazy regions; an improvement is needed to automate this process.

Step 2) Reconducting the clustering analysis for distinguishing different surface types from a subset of NIR bands. Because most aerosols are smaller than the NIR wavelengths, scattering in these bands is very weak. Also, avoid bands which are highly sensitive to absorbed atmospheric gases (water vapor).

Step 3) Assigning the mean value of each cluster in the clear region to the same cluster in the hazy region. Assuming that the same cluster in the clear hazy regions has similar reflectance values, the aerosol properties over the clear regions are assumed to be known (a visibility value of $30 \mathrm{~km}$ was used in this letter).

Step 4) Retrieving aerosol optical depths on a pixel basis using the lookup table method from at-sensor radiance and the assigned surface reflectance in a hazy region.

Step 5) Smoothing the aerosol optical depths spatially. This can reduce errors resultant from earlier assumptions (each cluster has the same reflectance in both hazy

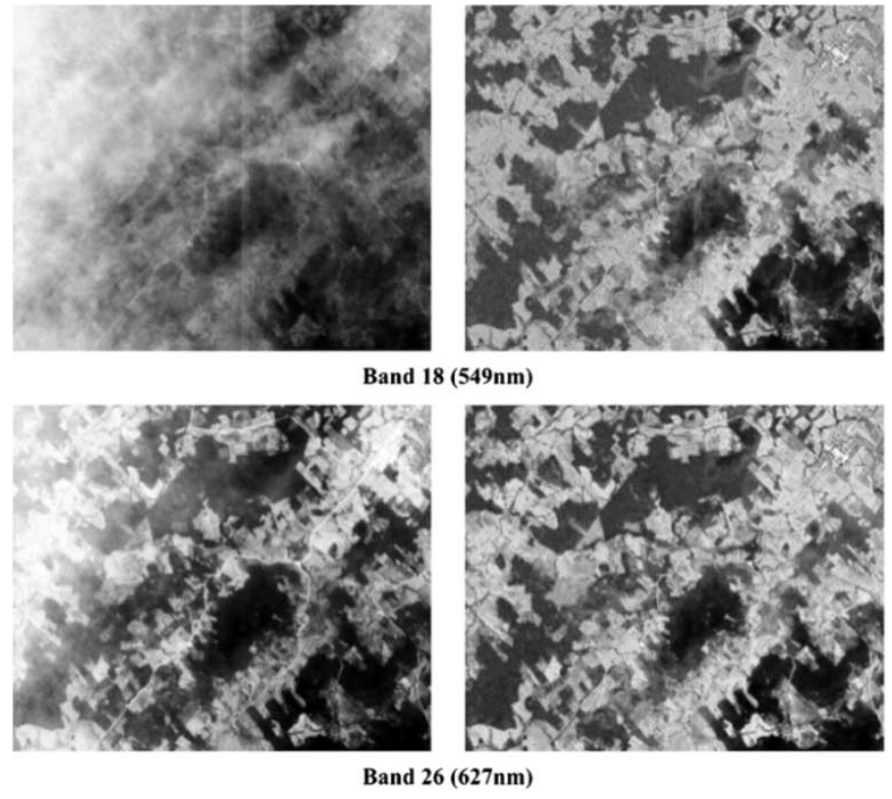

Fig. 1. (Left) Before and (right) after atmospheric correction of the AVIRIS imagery over Parana, Brazil, acquired on August 23, 1995 at two bands with the central wavelengths of 549 and $627 \mathrm{~nm}$.

and clear regions) and also reflects the fact that aerosols vary spatially much larger than the image pixel size.

Step 6) Determining the surface reflectance from at-sensor radiance and the smoothed aerosol optical depths using the lookup table method.

Because of AVIRIS' high spatial resolution, the surface adjacency effect also needs to be accounted for. The same analytic formulas from the original paper [9] also apply here. The lookup tables for atmospheric quantities are created from MODTRAN version 4 [22].

\section{B. Case Study}

Although an airborne system, AVIRIS images are often contaminated by horizontally inhomogeneous aerosols. The cluster matching technique was developed for ETM+ imagery [9] and tested over MODIS and SeaWiFS imagery [10], but has not been applied to hyperspectral imagery. Theoretically, it should work better with hyperspectral imagery since this algorithm relies, to a great extent, on spectral signatures. Use of shorter wavebands can be more effective in distinguishing hazy regions from clear, and the NIR bands can help distinguish different land cover types (clusters).

Fig. 1, AVIRIS imagery over Parana, was acquired on August 23,1995 . The central location is $11^{\circ} 13^{\prime} 24^{\prime \prime} \mathrm{S}$ by $61^{\circ} 17^{\prime} 27^{\prime \prime} \mathrm{W}$. The image is $512 \times 614$ with a $20-\mathrm{m}$ resolution. The left images, the originals, are heavily contaminated by smoke; the right images are corrected. The vertical lines in the image are due to AVIRIS instrument problems, and we did not attempt elimination. In this experiment, we predefined ten clusters using three visible bands $(10,14$, and 18) and 80 clusters using 15 NIR bands, every four bands from bands 42-98. More experiments may be needed to determine these bands optimally. If a cluster in the hazy regions does not have a correspondent in the clear 
region, no aerosol retrieval occurs, and its value is interpolated from the smoothing process. The water vapor contents are estimated using (3) and (4), which is discussed later. This extended algorithm works very well: most land features have been recovered on the upper left with smoke being removed.

\section{IMPROVED ALGORITHM FOR HYPERION}

The previous section demonstrated that cluster matching works quite well on AVIRIS imagery. However, it assumes that the same cluster between the test regions has the same visible reflectances; while required for multispectral imagery, this may not be necessary for hyperspectral imagery with the increased spectral information. The original algorithm water vapor content is not estimated from the imagery.

In this letter, we have provided two improvements over the original cluster matching technique: to revise Step 3) in the algorithm described in Section II-A; and to develop a new algorithm for estimating column water vapor content.

Hyperion, one of the three sensors on the NASA EO-1 platform [3], launched on November 24, 2000, now orbits $1 \mathrm{~min}$ behind Landsat 7. As a pushbroom imaging instrument, Hyperion provides high resolution hyperspectral images capable of resolving 220 spectral bands (from $0.4-2.5 \mu \mathrm{m}$ ) with a $10-\mathrm{nm}$ spectral resolution and a 30-m spatial resolution. The instrument covers $7.5 \times 100 \mathrm{~km}$ per image. Hyperion has a single telescope and two spectrometers: one for the visible and NIR region and another for the shortwave infrared region. The detail is available elsewhere [2].

\section{A. Improvement of Aerosol Estimation}

One major assumption in Section II-A is that a given cluster has the same reflectance in hazy and clear regions. It was appropriate for multispectral data (e.g., ETM+) because of the limited spectral information available. To recover the reflectance variability, we need to explore whether this assumption can be released with the rich spectral signatures of hyperspectral remote sensing. In this letter, the NIR band reflectances from the clear region are used to predict deviations from the mean value. The established relations are then applied to the hazy region to adjust the mean visible reflectance. Therefore, the improved procedure is identical to the old, except that the third step is replaced by the regression analysis for the reflectance deviation $\Delta r^{i}$ at visible band $i$ using $n$ NIR bands $\left(\mathrm{IR}^{1}, \ldots, \mathrm{IR}^{n}\right)$

$$
\Delta r^{i}=f_{i}\left(\mathrm{IR}^{1}, \mathrm{IR}^{2}, \ldots, \mathrm{IR}^{n}\right) .
$$

Thus, the reflectance of each pixel in the hazy region is the mean value $\left(\bar{r}^{i}\right)$ of each cluster from the clear region plus the weighted reflectance deviation predicted above

$$
\hat{r}^{i}=\bar{r}^{i}+\gamma_{i} \Delta r^{i}
$$

where $\gamma_{i}$ is the weighing factor between 0 and 1 . The purpose is to control the uncertainty of the prediction (1). If the fitting residual is very small, it is close to 1 ; otherwise, it is close to 0 . If $\gamma_{i}=0$, the improved method is equivalent to the original cluster matching algorithm.

The prediction function $f_{i}()$ may be linear or nonlinear, depending on the number of NIR bands used and the fitting resid- uals. Our experiments with Hyperion data showed that the linear relationships are sufficient with multiple NIR bands.

\section{B. Estimation of Column Water Vapor Content}

A number of algorithms, basically evaluating the 940-nm water absorption and relating that to the total column water content, have been developed to derive the column water vapor content of the atmosphere [1]. They include the narrowband and wideband ratio technique [23], continuum interpolated band ratio (CIBR) [24], [25], curve fitting method [26], the atmospheric precorrected differential absorption technique (APDA) [27], and the "smoothness test" approach in High Accuracy Atmospheric Correction for Hyperspectral Data (HATCH) [19], [20]. The widely used CIBR method has been adapted for deriving the water vapor content product from MODIS [25], [28], POLDER [29], [30], and the Modular Optoelectronic Scanner (MOS) [31]. Most algorithms, sensitive to variations in surface reflectance, particularly low-reflectance surfaces, require a precorrection of aerosol effects when the atmosphere is unclear.

Accurate estimation of water vapor content should take advantage of the large number of hyperspectral bands-most existing methods rely on only a few bands. These methods are certainly compact and easy to implement, but cannot consider factors such as variations in aerosol loading and surface reflectance. Most methods suggest that aerosol correction should occur before water vapor estimation. However, estimating aerosol loadings often requires a priori knowledge of water vapor content (e.g., the "dark-object" method). When more bands are used, the computational requirement increases. The algorithm we developed using multiple hyperspectral bands is very efficient in the operational process and does not require a precorrection of aerosol effects.

This algorithm is based on extensive MODTRAN simulations and the neural network method. Different atmospheric profiles from the Large Scale Biosphere-Atmosphere Experiment in Amazonia ( LBA) study, representing the tropical regions, from the Boreal Ecosystems Atmosphere Study (BOREAS) study, representing the middle-latitude regions and high-latitude U.S. Navy standard profiles, are input to MODTRAN 4; the integrated column water vapor content varies from $0.4-6.9 \mathrm{~cm}$. The MODTRAN simulations also incorporate surface reflectance spectra from the USGS spectra library [32], six aerosol visibility values $(5,10,15,20,35$, and $50 \mathrm{~km})$, and eight solar zenith angles. Simulated at-sensor radiance curves (around 0.94 and $1.13 \mu \mathrm{m}$ ) integrated over AVIRIS bands are displayed in Fig. 2. Lines at the bottom, middle, and top represent the results from LBA, BOREAS, and U.S. Navy profiles, respectively. The atmospheric visibility is $15 \mathrm{~km}$, and the surface is assumed grassy. AVIRIS band 62, a central wavelength of $0.943 \mu \mathrm{m}$, corresponds to the maximum absorption valley. Higher water vapor content corresponds to deeper valleys.

We first applied the CIBR method to estimation of the water vapor contents. Three AVIRIS bands, including band 56 $(885.83 \mathrm{~nm})$, band $62(943.42 \mathrm{~nm})$, and band $72(1039.48 \mathrm{~nm})$, were used to calculate the CIBR index

$$
\mathrm{CIBR}_{\text {AVIRIS }}=\frac{L(62)}{0.628^{*} L(56)+0.372^{*} L(72)} .
$$




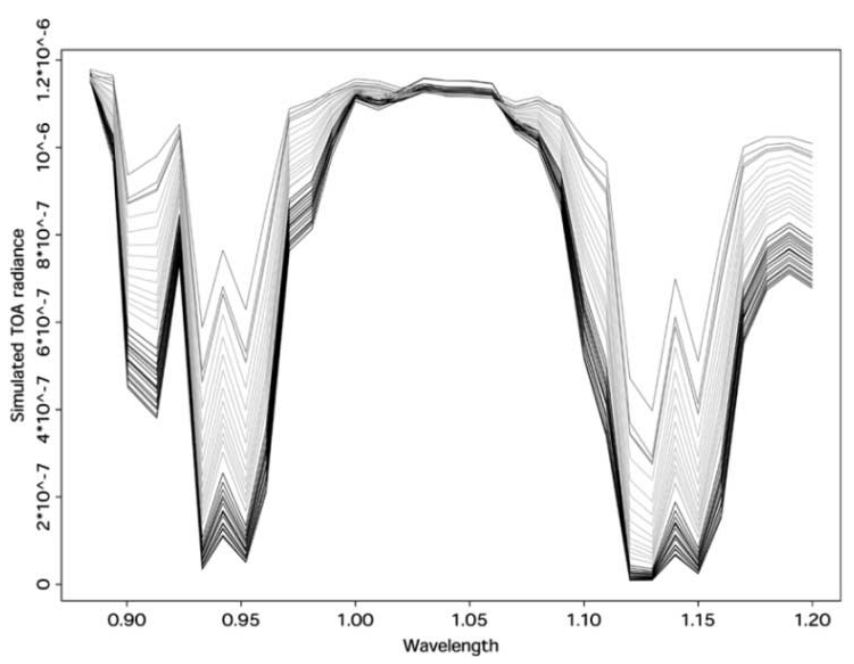

Fig. 2. Simulated top-of-atmosphere radiance spectra at AVIRIS bands (in wavelength, micrometers) with different water vapor contents of the atmosphere. Band 62 is close to the water vapor absorption region at about $940 \mathrm{~nm}$.

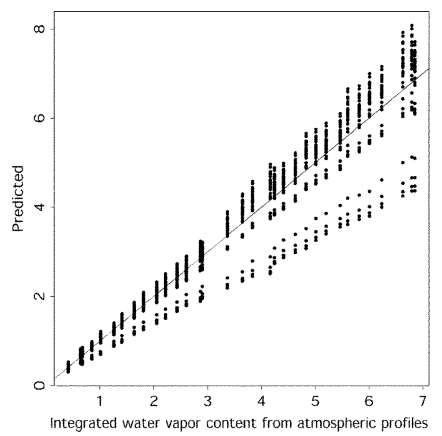

(a)

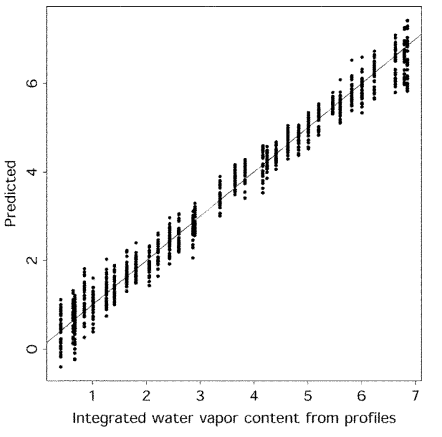

(b)
Fig. 3. Comparisons of the original integrated water vapor contents with the predicted water vapor contents from the simulated database as illustrated in Fig. 2 using (a) the CIBR algorithm and (b) the neural network algorithm.

$L(x)$ is the at-sensor radiance of band $\mathrm{X}$; the coefficients 0.628 and 0.372 are determined based on the relative distances of two bands from the central water absorption band (62). Bands 56 and 72 were selected because they do not seem to be significantly affected by water vapor absorption. Based on the simulation datasets with four aerosol loadings with the horizontal visibility values of $5,10,15$, and $20 \mathrm{~km}$ and eight surface reflectance spectra of different soils and canopies, we came out the following equation:

$$
\log \left(\mathrm{CIBR}_{\mathrm{AVIRIS}}\right)=-0.663545^{*} w^{0.6358}
$$

where $w$ is water vapor content in centimeters [predicted and original water vapor contents are compared in Fig. 3(a)]. The resulting correlation coefficient and the residual standard error (RSE) are $R^{2}=0.93$ and $\mathrm{RSE}=0.54$, respectively. It is quite clear that aerosol loadings and surface reflectances significantly affect the prediction.

Alternately, use the nonparametric regression technique. The artificial neural network algorithm [33] finds the best projection directions so that the primary variable will be enhanced and unwanted variables will be compressed. Use of the neural network is composed of two stages: training and prediction. The

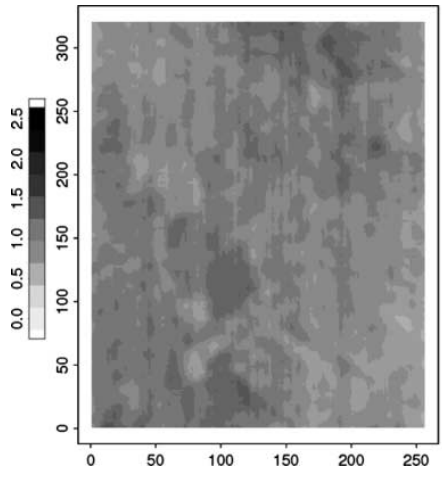

(a)

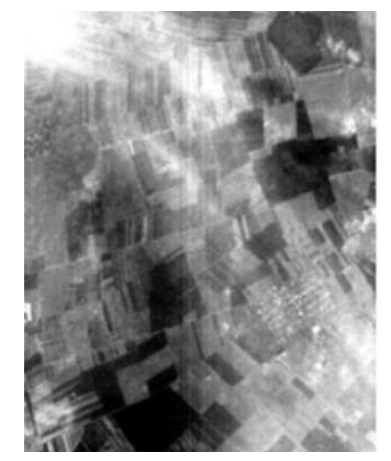

(c)

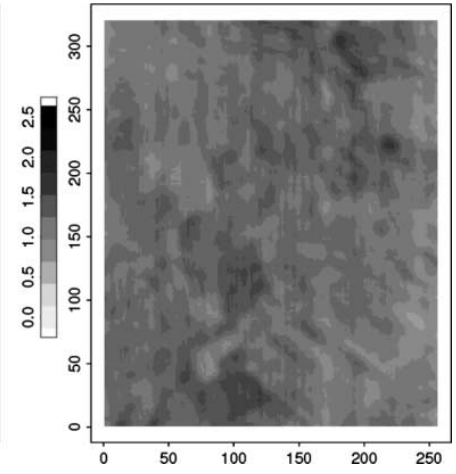

(b)

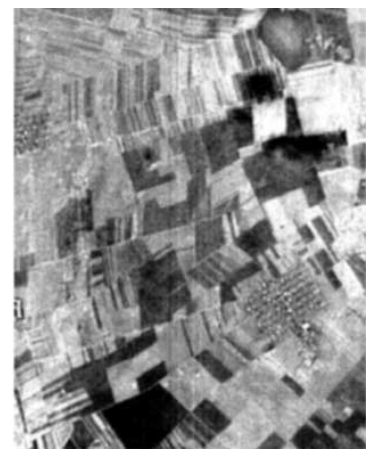

(d)
Fig. 4. (a) and (b) Water vapor content estimated from the Hyperion imagery acquired on May 18, 2001 over Bucharest, Romania using the CIBR algorithm (left) and the neural network (right) algorithm. (c) and (d) Band 12 Hyperion imagery (left) before and (right) after atmospheric correction.

results from the extensive MODTRAN simulations are used for training. Although very slow, training can be performed offline. The feed-forward neural network used in this letter is available from S-Plus software [34] and has only one hidden layer, but we can adjust the number of units in the hidden layer and another free parameter, the decay parameter. The fitted and the original water vapor content are shown in Fig. 3(b). The fitting is much better than the CIBR algorithm with a much higher correlation coefficient $\left(R^{2}=0.98\right)$ and smaller $\mathrm{RSE}=0.29$. Note that the prediction errors for low water vapor values are actually larger than those by the CIBR algorithm.

\section{Case Studies}

Two experiments were conducted using the Hyperion imagery acquired on May 18, 2001, near Bucharest, Romania, where the Fundulea Agricultural Station is located. The first ten bands contain no data (or the signal-to-noise ratios are too small) and were excluded. Bands 11 and 20 were used to identify the hazy regions out of ten clusters. The first image is 256 (pixels) by 320 (lines). The radiance values at the same spectral range observed by two spectrometers were calculated from their averages. The estimated water vapor contents using CIBR method and the neural network (with five units in the hidden layer) algorithm are shown in Fig. 4(a) and (b). The CIBR method was based on the fitted relationship with four Hyperion bands from the MODTRAN simulated dataset

$$
\log \left(\mathrm{CIBR}_{\text {Hyperion }}\right)=1.3606^{*} w^{0.3153}
$$


where

$$
\mathrm{CIBR}_{\text {Hyperion }}=\frac{[L(79)+L(80)]^{*} 0.5}{0.511^{*} L(52)+0.489 * L(86)} .
$$

Bands 52, 79, 80, and 86 have central wavelengths of 875.9 , 932.7, 942.8, and $1003.4 \mathrm{~nm}$. It is assumed that the central wavelengths are constant over all imagery. For the neural network algorithm, we first conducted tests using bands 56-62 and 77-83. Unfortunately, bands 56-62 are too noisy and only 77-83 were used. Because of the noisy data, all these bands were first smoothed using a $9 \times 9$ moving window determined from a series of experiments.

The water vapor map shows larger values in the diagonal direction from the upper right to the lower left. The predicted values at these corners, from both methods, are similar. The measured value from the nearby Sunphotometer is $1.534 \mathrm{~cm}$ and the average values of the retrieved map are 1.204 and 1.009 from the new method and the CIBR method, respectively. Both are very close. Since there is no ground measurement in more locations, it is hard to conclude whether the new algorithm is better just based on this experiment. More validation is needed. Fig. 4(c) and (d) shows the original Hyperion image and the corrected one. The differences are considerably significant. Since the water vapor mainly affects reflectance at the longer wavelengths, there are no significant impacts on the visible imagery. $f_{i}()$ in (1) are linear and the details are omitted here. Similar to the AVIRIS problem in Fig. 1, there are also vertical patterns; due to HYPERION sensor problems we did not attempt to eliminate them.

We have neglected the "smile" effect of the Hyperion sensor, an up-to-2.5-nm shift from the central pixel to the edge pixels [2]. A primary analysis indicates our approximation may result in an error of approximately $10 \%$ at the edge of the image for the water vapor retrieval. An ideal solution considers the "smile" effect on the pixel level. A practical approach to correcting this error divides the image into several regions and uses the averaged central wavelengths for each. Though the lookup tables are slightly different for each region, the basic approach is exactly the same.

Fig. 5 shows another example of the Hyperion imagery before and after atmospheric correction. The purpose is to demonstrate that this improved atmospheric correction method can work under different conditions. The image size is $256 \times 450$, extracted from the same Hyperion scene from which Fig. 4 was extracted. There are significant visual differences on the right side of the image, primarily due to aerosol removal. Most ground features have been recovered, but ground measurements are needed to quantify the correction accuracy.

\section{SUMMARY AND CONCLUSION}

Atmospheric correction is one key step for quantitatively estimating land surface variables from hyperspectral imagery. It is very challenging, especially when aerosol distribution is heterogeneous. In previous studies, we developed the cluster matching algorithm for ETM+ imagery and validated it using ground measurements. In this letter, we successfully adapted it to correct AVIRIS imagery and then improved this algorithm by releasing one primary assumption for aerosol estimation

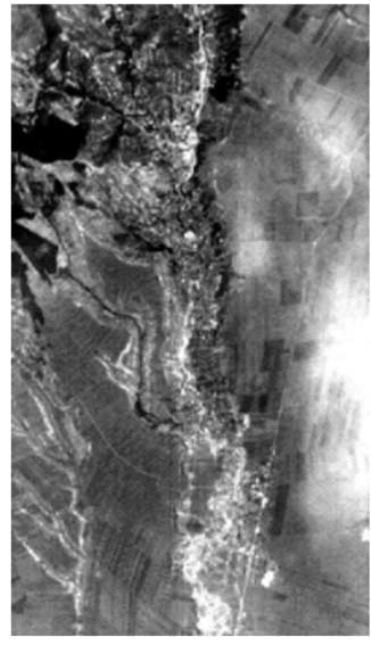

(a)

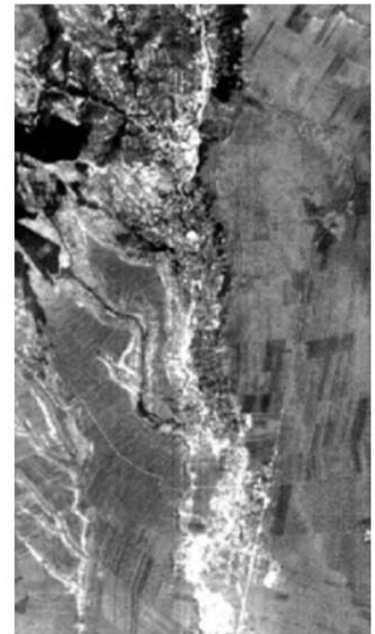

(b)
Fig. 5. Band 12 Hyperion imagery extracted from the same Hyperion scene as Fig. 4 (a) before and (b) after atmospheric correction.

and incorporated a new algorithm for column water vapor estimation. The improved algorithm performed very well over two Hyperion images.

This method is designed to correct heterogeneous aerosols, assuming there are one or more clear regions available, locally. It does not work where the horizontal distribution of aerosols is homogeneous. Another assumption is that the same clusters over the hazy regions can be found over the clear. The algorithm will fail if the cover types are completely different over both regions. These limitations, under most conditions, do not appear serious as long as the scene is sufficiently large.

There are still two other assumptions in the aerosol estimation algorithm: the known-aerosol model and the Lambertian surface. Further studies are needed to release these assumptions. For example, we may be able to identify the aerosol types by executing this algorithm for different aerosol models and then examining the spectral dependence of the retrieved aerosol optical depths. It might be feasible to couple this atmospheric correction algorithm with a set of simplified surface reflectance models (i.e., canopy, soil, snow, and water) [35]. Instead of estimating Lambertian surface reflectance, we may invert certain key parameters (e.g., leaf area index and spherical albedo) of the surface reflectance models that can eventually predict directional surface reflectances.

The newly developed neural network algorithm for estimating water vapor content is very promising since it is not sensitive to the variations in aerosol loadings and surface reflectance. The case studies using Hyperion data have indicated it works very well.

Note that these methods have not been validated using ground measurements. Further quantitative validation activities are needed before they can be implemented operationally.

\section{ACKNOWLEDGMENT}

The authors wish to thank S. Ungar, T. Brakke, L. Ong, and J. Pearlman for their leadership in the EO1 Project and assistance in the handling of Hyperion data. The authors are also grateful to $\mathrm{R}$. Green for providing AVIRIS data and 
G. Anderson and her group for making MODTRAN code public. The anonymous reviewers comments helped improve this letter's presentation.

\section{REFERENCES}

[1] R. O. Green, M. L. Eastwood, C. M. Sarture, T. G. Chrien, M. Aronsson, B. J. Chippendale, J. A. Faust, B. E. Pavri, C. J. Chovit, M. Solis, M. R. Olah, and O. Williams, "Imaging spectroscopy and the Airborne Visible/Infrared Imaging Spectrometer (AVIRIS)," Remote Sens. Environ., vol. 65, pp. 227-248, 1998.

[2] J. S. Pearlman, P. S. Barry, C. C. Segal, J. Shepanski, D. Beiso, and S. L. Carman, "Hyperion, a space-based imaging spectrometer," IEEE Trans. Geosci. Remote Sensing, vol. 41, pp. 1160-1173, June 2003.

[3] S. G. Ungar, J. S. Pearlman, J. A. Mendenhall, and D. Reuter, "Overview of the Earth Observing One (EO-1) mission," IEEE Trans. Geosci. Remote Sensing, vol. 41, pp. 1149-1159, June 2003.

[4] S. Liang, Quantitative Remote Sensing of Land Surfaces. New York: Wiley, 2003

[5] Y. J. Kaufman, "The atmospheric effect on remote sensing and its correction," in Theory and Applications of Optical Remote Sensing, G. Asrar, Ed. New York: Wiley, 1989, pp. 336-428

[6] D. Tanre and M. Legrand, "On the satellite retrieval of Saharan dust optical thickness over land: Two different approaches," J. Geophys. Res., vol. 96, pp. 5221-5227, 1991

[7] J. V. Martonchik, D. J. Diner, R. A. Kahn, T. P. Ackerman, M. E. Verstraete, B. Pinty, and H. R. Gordon, "Techniques for the retrieval of aerosol properties over land and ocean using multiangle imaging," IEEE Trans. Geosci. Remote Sensing, vol. 36, pp. 1212-1227, July 1998.

[8] R. Richter, "Correction of atmospheric and topographic effects for high spatial resolution satellite imagery," Int. J. Remote Sens., vol. 18, pp. 1099-1111, 1997

[9] S. Liang, H. Fang, and M. Chen, "Atmospheric correction of Landsat ETM+ land surface imagery: I. Methods," IEEE Trans. Geosci. Remote Sensing, vol. 39, pp. 2490-2498, Nov. 2001.

[10] S. Liang, H. Fang, M. Chen, C. Shuey, C. Walthall, and C. Daughtry, "Atmospheric correction of Landsat ETM+ land surface imagery: II. Validation and applications," IEEE Trans. Geosci. Remote Sensing, vol. 40, pp. 2736-2746, Dec. 2002

[11] Y. J. Kaufman, D. Tanré, L. Remer, E. F. Vermote, A. Chu, and B. N. Holben, "Operational remote sensing of tropospheric aerosol over the land from EOS-MODIS.," J. Geophys. Res., vol. 102, pp. $17051-17068,1997$.

[12] S. Liang, H. Fallah-Adl, S. Kalluri, J. JaJa, Y. J. Kaufman, and J. R. G. Townshend, "An operational atmospheric correction algorithm for Landsat Thematic Mapper imagery over the land," J. Geophys. Res., vol. 102, pp. 17173-17 186, 1997.

[13] P. M. Teillet and G. Fedosejevs, "On the dark target approach to atmospheric correction of remotely sensed data," Can. J. Remote Sens., vol. 21, pp. 374-387, 1995.

[14] J. V. Martonchik, D. J. Diner, K. A. Crean, and M. A. Bull, "Regional aerosol retrieval results from MISR," IEEE Trans. Geosci. Remote Sensing, vol. 40, pp. 1520-1531, July 2002.

[15] P. R. J. North, "Estimation of aerosol opacity and land surface bidirectional reflectance from ATSR-2 dual-angle imagery: Operational method and validation," J. Geophys. Res., vol. 107, no. 4149, 2002.

[16] J. Zhang and S. A. Christopher, "Intercomparison of smoke aerosol optical thickness derived from GORS 8 imager and ground-based sun photometers," J. Geophys. Res., vol. 106, pp. 7387-7397, 2001.

[17] S. Liang and H. Fang, A new atmospheric correction algorithm for MODIS land surface imagery, in Remote Sens. Environ., 2004, submitted for publication.
[18] J. L. Deuze, F. M. Breon, C. Devaux, P. Goloub, M. Herman, B. Lafrance, F. Maignan, A. Marchand, F. Nadal, G. Perry, and D. Tanre, "Remote sensing of aerosols over land surfaces from POLDER-ADEOS-1 polarized measurements," J. Geophys. Res., vol. 106, pp. 4913-4926, 2001.

[19] Z. Qu, B. C. Kindel, and A. F. H. Goetz, "The High Accuracy Atmospheric Correction for Hyperspectral Data $(\mathrm{HATCH})$ model," IEEE Trans. Geosci. Remote Sensing, vol. 41, pp. 1223-1231, June 2003.

[20] A. F. H. Goetz, B. C. Kindel, M. Ferri, and Z. Qu, "HATCH: Results from simulated radiances, AVIRIS and Hyperion," IEEE Trans. Geosci. Remote Sensing, vol. 41, pp. 1215-1222, June 2003.

[21] M. W. Matthew, S. M. Adler-Golden, A. Berk, S. C. Richtsmeier, R. Y. Levin, L. S. Bernstein, P. K. Acharya, G. P. Anderson, G. W. Felde, M. P. Hoke, A. Ratkowski, H. H. Burke, R. D. Kaiser, and D. P. Miller, "Status of atmospheric correction using a MODTRAN4-based algorithm," Proc. SPIE, vol. 4049, pp. 199-207, 2000.

[22] A. Berk, L. S. Bernstein, G. P. Anderson, P. K. Acharya, D. C. Robertson, J. H. Chetwynd, and S. M. A. Golden, "MODTRAN cloud and multiple scattering upgrades with application to AVIRIS," Remote Sens. Environ. vol. 65 , pp. 367-375, 1998 .

[23] R. Frouin, P. Y. Deschamps, and P. Lecomte, "Determination from space of atmospheric total water vapor amounts by differential absorption near $940 \mathrm{~nm}$ : Theory and airborne verification," J. Appl. Meteorol., vol. 29, pp. 448-459, 1990.

[24] C. J. Bruegge, J. E. Conel, R. O. Green, J. S. Margolis, R. G. Holm, and G. Toon, "Water-vapor column abundance retrievals during fife," $J$. Geophys. Res., vol. 97, pp. 18759-18768, 1992.

[25] Y. Kaufman and B. C. Gao, "Remote sensing of water vapor in the near IR from EOS/MODIS," IEEE Trans. Geosci. Remote Sensing, vol. 30, pp. 871-884, Sept. 1992

[26] B. C. Gao and A. Goetz, "Determination of total column water vapor in the atmosphere at high spatial resolution from AVIRIS data using spectral curve fitting and band ratioing techniques," Proc. SPIE, vol. 1298, pp. 138-149, 1990.

[27] D. Schlapfer, C. C. Borel, J. Keller, and K. I. Itten, "Atmospheric precorrected differential absorption technique to retrieve columnar water vapor," Remote Sens. Environ., vol. 65, pp. 353-366, 1998.

[28] M. D. King, W. P. Menzel, Y. J. Kaufman, D. Tanre, B. C. Gao, S. Platnick, S. A. Ackerman, L. A. Remer, R. Pincus, and P. A. Hubanks, "Cloud and aerosol properties, precipitable water, and profiles of temperature and water vapor from MODIS," IEEE Trans. Geosci. Remote Sensing, vol. 41, pp. 442-458, Feb. 2003

[29] M. Vesperini, F. M. Breon, and D. Tanre, "Atmospheric water vapor content from spaceborne POLDER measurements," IEEE Trans. Geosci. Remote Sensing, vol. 37, pp. 1613-1619, May 1999.

[30] S. Bouffies, F. M. Breon, D. Tanre, and P. Dubuisson, "Atmospheric water vapor estimate by a differential absorption technique with the POLDER instrument," J. Geophys. Res., vol. 102, pp. 3831-3841, 1997.

[31] S. Tahl and M. V. Schonermark, "Determination of the column water vapor of the atmosphere using backscattered solar radiation measured by the Modular Optoelectronic Scanner (MOS)," Int. J. Remote Sens., vol. 19, pp. 3223-3236, 1998.

[32] R. N. Clark, G. A. Swayze, A. J. Gallagher, T. V. V. King, and W. M. Calvin, "The U.S. Geological Survey digital spectral library: Version 1: 0.2 to 3.0 microns," USGS, Washington, DC, USGS Open File Rep. 93-592, 1993

[33] B. D. Ripley, "Neural networks and flexible regression and discrimination," Adv. Appl. Statist., vol. 2, pp. 39-57, 1994.

[34] W. N. Venables and B. D. Ripley, Modern Applied Statistics with S-Plus. New York: Springer-Verlag, 1994.

[35] S. Liang and A. H. Strahler, "Land surface bidirectional reflectance distribution function (BRDF): Recent advances and future prospects," in Remote Sens. Rev., 2000, vol. 18, pp. 83-551. 